\title{
Aplicación del modelo meteorológico WRF para el pronóstico de precipitaciones en período lluvioso de Cuba, 2014
}

\author{
Application of the WRF meteorological model for rainfall \\ forecast in the Cuba rainy season, 2014
}

\author{
Aldo S. Moya Álvarez' \\ Universidad Continental, Perú \\ aldomoya00@gmail.com
}

\author{
José M. Ortega León² \\ Centro Meteorológico de Villa Clara, Cuba
}

\section{RESUMEN}

El objetivo ha sido la aplicación del modelo meteorológico WRF y verificar su pronóstico de precipitaciones para Cuba en el período lluvioso del año 2014, para lo cual se han construido dos dominios, uno externo de $24 \times 24 \mathrm{~km}$ y otro interno de $8 \times 8 \mathrm{~km}$ de resolución. Algunos de los esquemas de parametrización empleados fueron el ACM2 para capa fronteriza y el de Kain-Fritsch para la convección. Se evaluó el pronóstico de precipitaciones desde 6 hasta 42 horas. La verificación se realizó con ayuda de los datos de la Red de Estaciones Meteorológicas de Cuba. Se emplearon dos métodos, uno basado en la verificación puntual para el pronóstico cuantitativo, y otro conocido como "verificación parcial", utilizado para el pronóstico alternativo. Como resultados se lograron la implementación del modelo WRF y su verificación, la cual determinó que este modelo subestima las magnitudes de las precipitaciones, aunque las desviaciones no superan los $5 \mathrm{~mm}$ respecto al real en las tardes. Desde el punto de vista de ocurrencia o no del evento "lluvia" el modelo también subestima, aunque logra niveles de detección elevados, $81 \%$ para el plazo de pronóstico 06-12 horas y $73 \%$ para el plazo 30-36 horas. Se concluye que el modelo WRF subestima ligeramente la magnitud de las precipitaciones, pero logra niveles elevados de detección de las mismas, por lo que resulta de gran utilidad para la realización de los pronósticos de lluvias.

Palabras clave: Modelo meteorológico, aplicación, verificación, período lluvioso.

\begin{abstract}
The objective was the application of the WRF meteorological model and verify its rainfall forecast in the Cuba rainy season in 2014, for that two domains were built, one external of $24 \times 24 \mathrm{~km}$ and one internal of $8 \times 8 \mathrm{~km}$ resolution. Some of the used parameterization schemes were the ACM2 for boundary layer and the Kain-Fritsch for the convection. Rainfall forecasting was evaluated from 6 to 42 hours. The verification was performed using data from the Meteorological Station Network in Cuba. Two methods were used, one based on the punctual verification for the quantitative forecast, and another known as "partial verification" used for alternative forecast. As results, the WRF model implementation and its verification are achieved, which determined that this model underestimates the rainfall magnitudes, although deviations don't overcome the $5 \mathrm{~mm}$ respect to the real one in the afternoons. From the occurrence viewpoint or not of the "rain" event the model also underestimates, but achieves high detection levels, $81 \%$ for the forecast term 06-12 hours and $73 \%$ for the term 30-36 hours. We conclude that the WRF model slightly underestimates the rainfall magnitude, but achieves high detection levels, which is really useful for making rain forecasts.
\end{abstract}

Keywords: Meteorological model, application, verification, rainy season.

Historial del artículo:

Recibido: 24 de marzo de 2015. Aprobado: 09 de mayo 2015. Disponible en línea: 30 de junio de 2015

1 Ingeniero Meteorólogo; Doctor en Ciencias Meteorológicas; Jefe de Pronósticos de Villa Clara, Cuba; asesor científico en la Universidad Continental, Perú.

2 Licenciado en Ciencias de la Computación del Centro Meteorológico de Villa Clara, Cuba. 


\section{INTRODUCCIÓN}

El modelo meteorológico WRF (Weather Research and Forecasting) es un modelo de última generación, que se ha convertido en el más utilizado en el mundo durante los últimos años para el pronóstico de campos de variables meteorológicas a escala regional. Las características principales del modelo WRF giran alrededor de su dinámica no hidrostática y de su capacidad de permitir el uso de resoluciones espaciales de unos pocos kilómetros. Da la posibilidad de varias opciones de configuración y de parametrizaciones físicas para la convección, la Capa Fronteriza Planetaria y la radiación, entre otros procesos hidrotermodinámicos. Su desarrollo comenzó en la última parte de la década de 1990 y fue una asociación de colaboración principalmente entre el Centro Nacional de Investigación Atmosférica (NCAR), la Administración Nacional Oceánica y Atmosférica, la Agencia de Tiempo de la Fuerza Aérea (AFWA), el Laboratorio de Investigación Naval, la Universidad de Oklahoma y la Administración Federal de Aviación (FAA), todos de los Estados Unidos de América. El modelo ofrece una plataforma de pronóstico flexible y eficiente, al tiempo que proporciona los últimos avances en la física y de los métodos numéricos y de asimilación de datos (1). Según Klemp (2), uno de los objetivos del WRF es acelerar los avances en las investigaciones relacionadas con la meteorología operativa a nivel mundial. El modelo brinda la posibilidad de ser utilizado para las simulaciones de variables y fenómenos meteorológicos en cualquier región del planeta.

Los primeros trabajos en Cuba dirigidos a la aplicación de modelos de alta resolución no hidrostáticos se deben a las investigaciones de Mitrani (3), que adaptó el modelo MM5 (Modelo Mesoescaslar de Quinta Generación) para el archipiélago cubano y mares adyacentes, el que se mantiene de forma operativa hasta la actualidad en el pronóstico de los campos de variables meteorológicas y de precipitaciones. Turtos et al. (4) evaluaron la implementación del modelo WRF en Cuba con el objetivo de diagnosticar la calidad del aire.

Las precipitaciones convectivas constituyen el fenómeno meteorológico más común en Cuba durante todo el año, pero cobra particular importancia en el período lluvioso, que se extiende entre los meses de mayo y octubre. Estas precipitaciones realizan un aporte significativo al régimen anual de las mismas en el archipiélago cubano, por lo que su pronóstico es de vital importancia, a la vez que, es aún en la actualidad, una tarea compleja para los pronosticadores de todo el país.

El presente trabajo tiene como objetivo fundamental evaluar los resultados del pronóstico de precipitaciones del modelo WRF (V. 3.5) en Cuba durante el período lluvioso de 2014, concretamente entre el 1 de mayo y el 15 de octubre. En este caso el papel fundamental dentro de las posibilidades de parametrización que brinda el modelo, lo juegan las parametrizaciones de cúmulos y de la capa fronteriza de la atmósfera. Por ejemplo, Wisse y Vila'-Guerau de Arellano (5) demostraron que los esquemas de parametrización de la capa fronteriza tienen un gran impacto en los campos de lluvia simulados, a partir de sus experiencias con el modelo MM5

\section{MATERIAL Y MÉTODOS}

El trabajo constituye una investigación aplicada y sus resultados se encuentran disponibles en el Servicio Meteorológico Cubano.

Para la implementación del modelo se emplearon las condiciones iniciales y de frontera del modelo meteorológico global GFS (Global Forecast System), con una resolución horizontal de 05 y limitada entre los 50 y los 110 de longitud oeste y entre el Ecuador y los 50 de latitud norte.

Se emplean 24 niveles de salida del GFS en el plano vertical (Nivel medio del mar (nmm), 1000, 975, 950, $925,900,850,800,750,700,650,600,550,500$, $450,400,350,300,250,200,150,100,70$, y 50 $\mathrm{hPa}$ ). Las variables tomadas fueron las siguientes:

En la altura: Altura de geopotencial, humedad relativa, temperatura, componentes zonal y meridional del viento.

En la superficie: Presión a nivel medio del mar, presión en la superficie, temperatura superficial, contenido de agua del suelo en 4 capas, temperatura y punto de rocío a 2 metros de altura, componentes zonal y meridional del viento a $10 \mathrm{~m}$ de altura y Albedo.

\section{Configuración del modelo WRF}

Las simulaciones se realizaron para dos dominios, mostrados en la figura 1. Las características de ambos se especifican en la tabla 1 ; el externo, de $24 \mathrm{~km}$ de paso de malla $(0,22$ aproximadamente), y el interno, anidado en el anterior, de $8 \mathrm{~km} \quad(0,07$ aproximadamente).

Como se indica en la tabla $\mathrm{N}^{\circ} 1$, las condiciones iniciales $y$ de contorno del dominio externo son tomadas del pronóstico del modelo global GFS con una resolución de 0,5, inicializado en el mismo horario para el cual se realizan las simulaciones de WRF. Las condiciones iniciales y de contorno del dominio interno son tomadas del pronóstico del dominio externo del WRF con una resolución de 24 km. 
Los esquemas de parametrización utilizados se muestran en la tabla 2. Para la convección se seleccionó el esquema de Kain-Fritsch, empleado ya

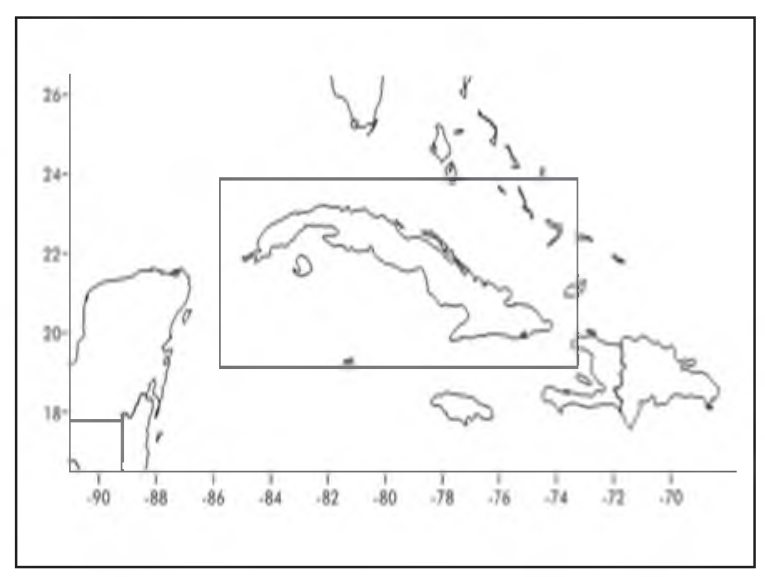

Figura $\mathrm{N}^{\circ}$ 1: Principales característica de los dominios y datos iniciales y de contorno.

Tabla $N^{\circ}$ 1: Principales característica de los dominios y datos iniciales y de contorno.

\begin{tabular}{|c|c|c|}
\hline Características & $\begin{array}{l}\text { Dominio } \\
\text { externo }\end{array}$ & $\begin{array}{l}\text { Dominio } \\
\text { interno }\end{array}$ \\
\hline $\begin{array}{l}\text { Paso horizontal de } \\
\text { malla }\end{array}$ & $24 \mathrm{~km}$ & $8 \mathrm{~km}$ \\
\hline Dimensiones ( $X Y Z$ ) & $55 \times 23 \times 40$ & $118 \times 41 \times 40$ \\
\hline Paso de tiempo & $180 \mathrm{~s}$ & $180 s$ \\
\hline Condiciones iniciales & $\begin{array}{l}\text { GFS } 0.5 \times 0.5 \\
\text { pronóstico }\end{array}$ & $\begin{array}{l}\text { Pronóstico de WRF } \\
24 \times 24 \mathrm{~km}\end{array}$ \\
\hline $\begin{array}{l}\text { Condiciones de } \\
\text { contorno (CC) }\end{array}$ & $\begin{array}{l}\text { GFS } 0,5 \times 0,5 \\
\text { pronóstico }\end{array}$ & $\begin{array}{c}\text { Pronóstico de WRF } \\
24 \times 24 \mathrm{~km}\end{array}$ \\
\hline Frecuencia de CC & 3 horas & $180 \mathrm{~s}$ \\
\hline
\end{tabular}

con buenos resultados en los trabajos de Mercader et al (6). Otros esquemas de parametrizaciones que se describen en la literatura internacional son los de Kuo (7 y 8), de Betts y Miller (9), el de Arakawa y Schubert (10) y el de Grell y Devenyi (11), entre otros.

Para la capa fronteriza de la atmósfera se seleccionó el modelo ACM2 (Asymmetric Convective Model 2), descrito en los trabajos de Pleim (12).

El modelo de radiación seleccionado fue el RRTMG (Rapid Radiative Transfer Model (13), que es una versión del RRTM más reciente y con una mejor representación de la nubosidad, no resuelta por la malla del modelo. El RRTM como tal fue descrito en Mlawer et al (14) y utiliza el método k-correlacionado (15) para implementar un algoritmo que destaca frente a otros por su rapidez. Está heredado del modelo MM5, antecesor de WRF

Para la microfísica se escogió el esquema de Lin et al
(16). Esta parametrización incluye el granizo blando, la sedimentación de hielo y el tiempo de disgregación del hielo.

El modelo de suelo es el llamado modelo "Noah LSM"; $^{\prime \prime}$ el Noah Land Surface Model (LSM) está basado en el "Oregon State University (OSU) LSM", que fue desarrollado por Pan y Mahrt (17) y por Chen et al (18). Comparando con datos del experimento FIFE, Chen et al (19) encontraron que el OSU LSM se comportaba a la altura de modelos más complejos. Su balance entre simplicidad y error, lo llevaron a ser implementado en el modelo operacional del NCEP ETA y en el modelo MM5 de NCAR. En Chen y Dudhia $(20,21)$ puede encontrarse una descripción de la implementación del OSU LSM en el MM5. El llamado Noah LSM es la evolución del OSU LSM implementada en WRF y en el "North American Mesoscale Model (NAM)". Este modelo divide el suelo en 4 capas de grosores: $10 \mathrm{~cm}$, $30 \mathrm{~cm}, 60 \mathrm{~cm}$ y $100 \mathrm{~cm}$, hasta una profundidad total de dos $m$.

Tabla N $N^{\circ}$ 2: Esquemas de parametrizaciones empleados.

\begin{tabular}{ll}
\hline Procesos & $\begin{array}{c}\text { Esquemas de parametrizaciones } \\
\text { (ambos dominios) }\end{array}$ \\
\hline Convección & Esquema Kain-Fritsch (new Eta). \\
Capa fronteriza & ACM2 \\
Microfísica & Esquema de Lin et al. \\
Radiación & Esquema RRTMG. \\
Suelo & Modelo de 4 capas de suelo. \\
Técnicas de & Retroalimentación en dos \\
anidamiento & direcciones (2-way nesting) \\
\hline
\end{tabular}

Período de simulación y de verificación de los resultados del pronóstico de precipitaciones

Las simulaciones y verificación del pronóstico de precipitaciones se realizaron para el período lluvioso del año 2014, solamente para el dominio interno. El período lluvioso se consideró entre el 1 de mayo y el 15 de octubre. Como datos de comparación se emplearon las precipitaciones en seis horas de la Red de Estaciones del Sistema Meteorológico Nacional. La verificación se hizo para el pronóstico de precipitaciones en 6 horas a partir de la inicialización de las 12 UTC (Tiempo Universal Coordinado) y se realizó mediante el empleo de varios índices estadísticos, obtenidos mediante la comparación del campo de lluvia pronosticado con el campo observado. La figura 2 representa la distribución espacial de la Red de Estaciones Meteorológicas del país.

El primer paso consistió en realizar un análisis objetivo 
de las precipitaciones observadas en la Red de Estaciones Meteorológicas de Cuba y llevarlo a una malla de $118 \times 41$ nodos, idéntica al campo de lluvia pronosticado. El método de interpolación aplicado fue el método de Shepard (22). Teniendo presente que solo se cuenta con datos de precipitaciones sobre el archipiélago, fue necesario aplicar una máscara a ambos campos para comparar solamente el área correspondiente al archipiélago cubano.

Con los campos obtenidos al aplicar la máscara se evaluaron los pronósticos de precipitación, atendiendo a dos variantes:

Verificación cuantitativa puntual, basada en la comparación punto a punto de los campos pronosticado y observado y verificación parcial (más conocida como fuzzy) que es flexible desde el punto de vista espacial y considera que un pronóstico también puede ser útil si se encuentra ligeramente desplazado
Donde:

$D(i, j)$ y $D_{a b s}(i, i)$ - Desviaciones media y media absoluta respectivamente en los nodos (i,i) de la malla.

$P_{F}(i, j), P_{A}(i, j)$ - Precipitación, pronóstico y análisis respectivamente en cada nodo $(i, i)$ de la malla.

$N_{i}$ y $N_{i}$ - Dimensiones y de la malla, respectivamente. La técnica de verificación parcial se basa en el criterio conocido como "mínima cobertura" (23), la cual consiste en considerar que el pronóstico es correcto si en una ventana de $A \times B$, puntos de malla, se pronostica $y$ es observado el suceso "lluvia" al menos en uno de los puntos. En nuestro caso las ventanas fueron construidas de $3 \times 3$ puntos $(16 \times 16 \mathrm{~km})$. Siguiendo el criterio anterior se construyeron tablas de contingencia (Tabla 3), en las que cada elemento de la matriz contiene el número de ocurrencias para las cuales las observaciones y el modelo alcanzan o exceden un

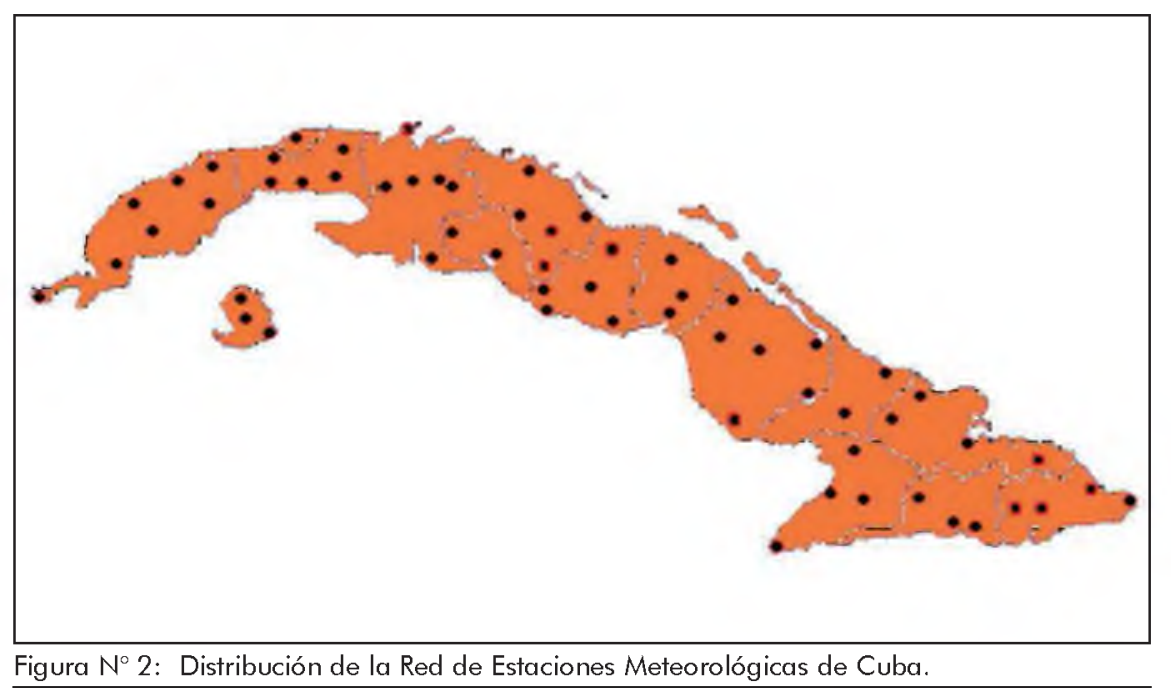

respecto a la observación en una ventana de puntos de la malla definido previamente $(6,23)$.

La técnica de verificación cuantitativa se basa en la comparación punto a punto entre el pronóstico y el real. En este caso, la verificación se realizó con ayuda de las fórmulas 1 (error medio absoluto, AME) y 2 (error medio, ME). El error medio (desviación media) permite definir el "sesgo" del modelo (24) y la desviación absoluta media informa sobre la magnitud del error del pronóstico respecto a la observación.

$$
\begin{aligned}
& D_{\text {abs }}(i, i)=\frac{1}{N_{i} N_{i}} \sum_{i} \sum_{1} \mid\left[P_{F}(i, i)-P_{A}(i, i)\right] \\
& D(i, i)=\frac{1}{N_{i} N_{i}} \sum_{i} \sum_{i}\left[P_{F}(i, i)-P_{A}(i, i)\right]
\end{aligned}
$$

umbral de precipitación para un período de pronóstico dado (23). En este caso se escogió el umbral 0.5 $\mathrm{mm}$ como umbral de ocurrencia de precipitación. A partir de las correspondencias entre ventanas se han calculado los índices que comúnmente se emplean en la verificación clásica punto a punto (Probability of detection, POD; False alarm ratio, FAR; Multiplicative, BIAS (sesgo); Success ratio, SR; etc).

Tabla N ${ }^{\circ}$ 3: Tabla de contingencia, donde cada elemento de la matriz contiene el número de casos en los que las observaciones y el modelo alcanzan - exceden un umbral de precipitación para un periodo de pronóstico dado.

\begin{tabular}{lcc}
\hline & \multicolumn{2}{c}{ Tabla de contingencia } \\
\hline Contingencia & Observado sí & Observado no \\
Pronóstico sí & Hits & False alarms \\
Pronóstico no & Misses & Correct negatives \\
\hline
\end{tabular}


Tabla $N^{\circ}$ 3: Tabla de contingencia, donde cada elemento de la matriz contiene el número de casos en los que las observaciones y el modelo alcanzan o exceden un umbral de precipitación para un periodo de pronóstico dado.

El índice "POD" (Probability of detection (hit rate)) indica en qué medida la lluvia observada fue

\section{RESULTADOS}

Como resultado de la implementación del modelo se cuenta hoy en Cuba con una versión operativa de WRF disponible a todos los pronosticadores del país y de regiones vecinas. Los resultados se visualizan a través del Sitio Web del Centro Meteorológico de Villa Clara

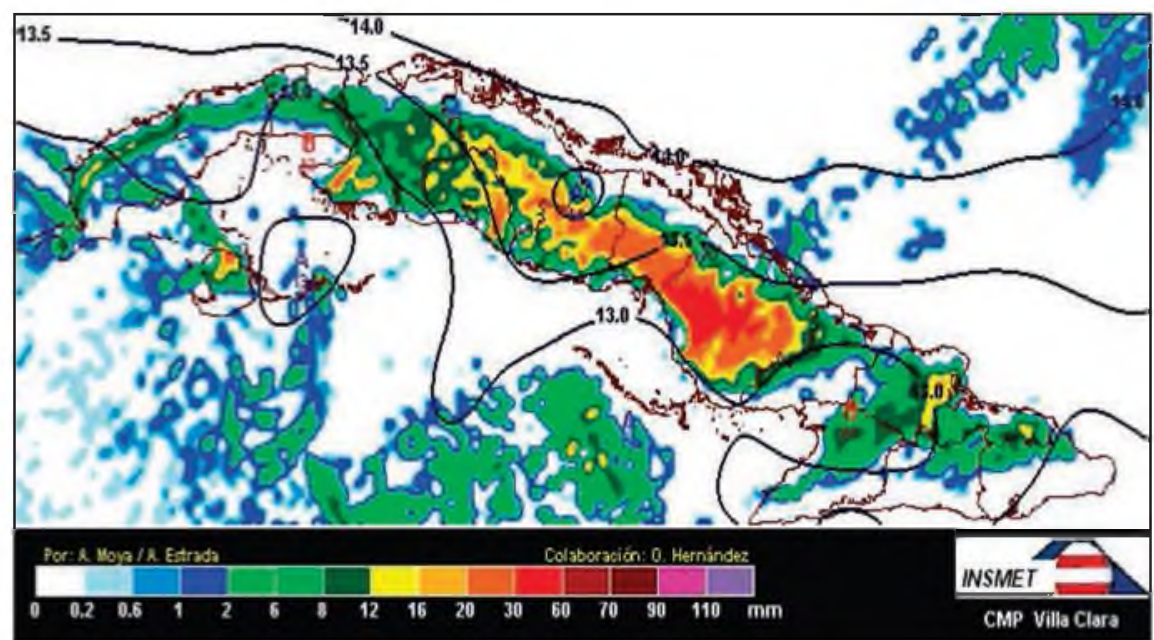

Figura $N^{\circ}$ 3: Campo de precipitaciones (acumulado en 3 horas) para el dominio interno, plazo 21 horas de la inicialización de las 00 UTC, válido para el período correspondiente entre las 2 y las 5 de la tarde.

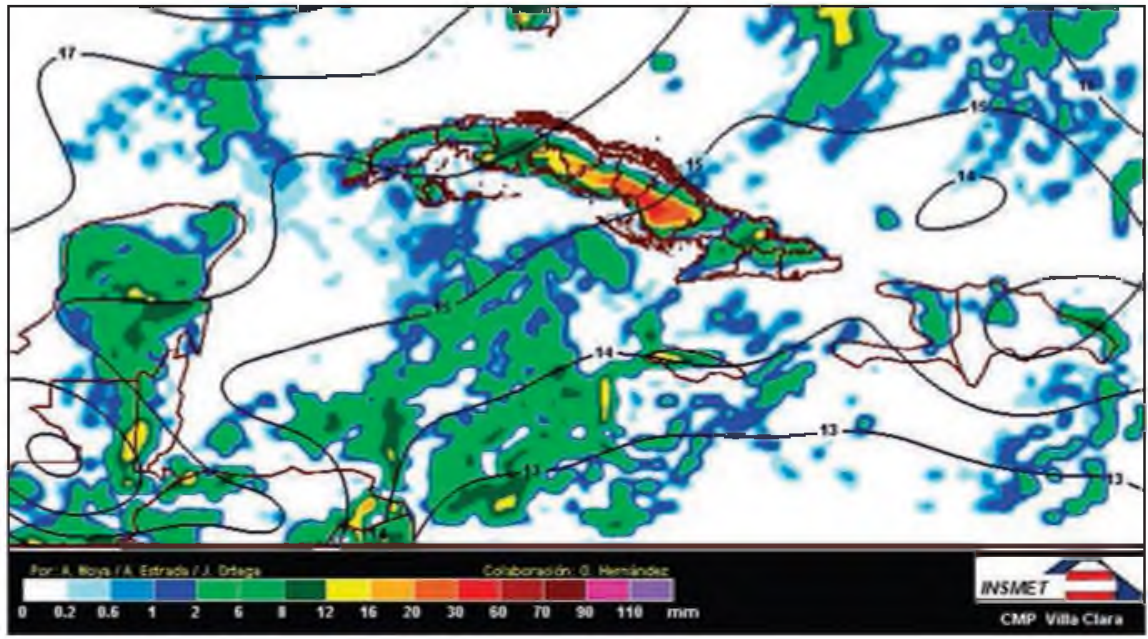

Figura $N^{\circ}$ 4: Campo de precipitaciones (acumulado en 3 horas) para el dominio externo, plazo 21 horas de la inicialización de las 00 UTC, válido para el período correspondiente entre las 2 y las 5 de la tarde.

pronosticada correctamente. El índice SR (Success ratio) indica la medida en que las lluvias pronosticadas fueron observadas. El índice FAR (False alarm ratio) indica en qué medida se pronósticaron eventos de precipitaciones y estos no ocurrieron y las BIAS indican en que medida el modelo sobreestimó o subestimó la ocurrencia de precipitaciones.
(25). Las corridas se realizan dos veces al día, para la inicialización de las 00 y de las 12 UTC. Se muestran los dos dominios descritos anteriormente y los campos de las variables meteorológicas se representan en forma de mapas, con bandas de colores e isolíneas, que describen la distribución espacial de las variables. Este trabajo se refiere solamente al pronóstico de los campos de precipitaciones. En ambas inicializaciones 
el plazo de pronóstico se extiende hasta las 72 horas. Las figuras $\mathrm{N}^{\circ} 3$ y $\mathrm{N}^{\circ} 4$ muestran las salidas de los campos de precipitaciones (acumulado en 3 horas) para los dominios interno (3) y externo (4) del modelo. Las figuras muestran el pronóstico correspondiente a un plazo de 21 horas a partir de la inicialización de las 00 UTC, válido para el período correspondiente entre las 2 y las 5 de la tarde en ambos casos. La característica principal del dominio interno, que lo meteorológico para una región más extensa, lo que pemite analizar el campo de precipitaciones de una manera más integral, aunque con menor nivel de detalles.

\section{Verificación puntual}

La figura $\mathrm{N}^{\circ} 5$ muestra el campo de la desviación

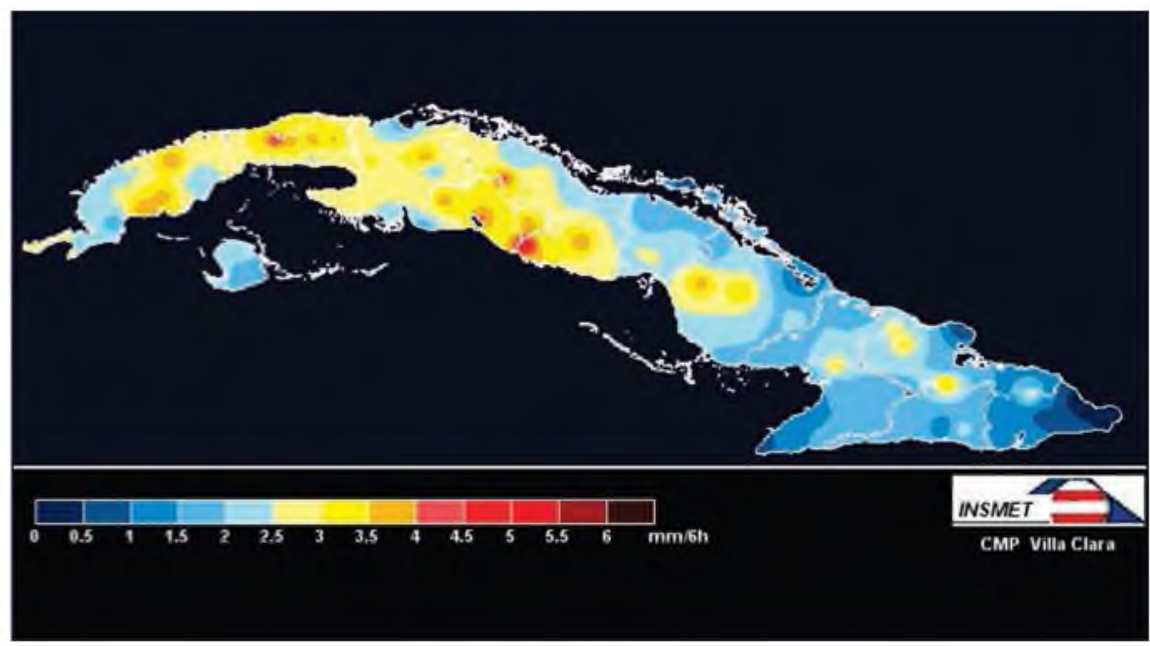

Figura $N^{\circ}$ 5: Campo de la desviación absoluta media del pronóstico para 30 - 36 horas (tarde del segundo día de pronóstico) respecto al real.

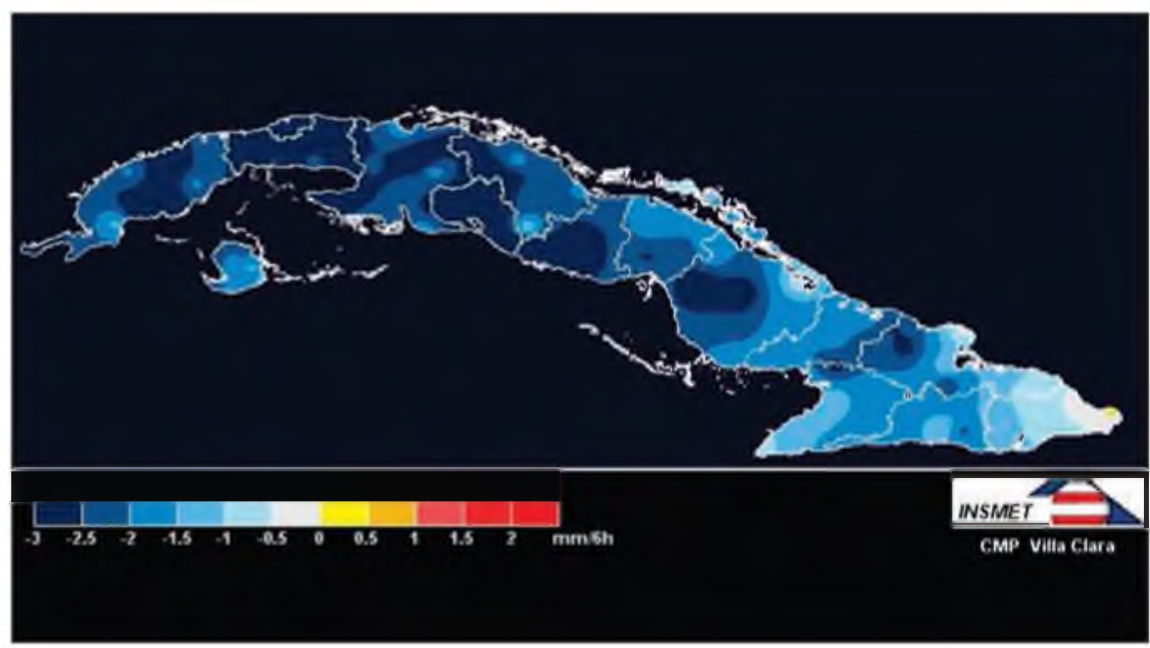

Figura $N^{\circ}$ 6: Campo de la desviación media (sesgo) del pronóstico para 30 - 36 horas (tarde del segundo día de pronóstico) respecto al real.

distingue del externo, es que refleja mucho más detalladamente las características físico geográficas de la región, lo que se hace más notable en zonas de orografía compleja. En este caso, el campo de precipitaciones esperado se puede apreciar con mayor claridad en el dominio interno y ello permite al pronosticador, realizar el pronóstico de precipitaciones con mayor nivel de precisión. La ventaja del dominio externo radica en que se puede apreciar el proceso media absoluta del pronóstico para el plazo 30 - 36 horas respecto al real. Este plazo se corresponde con el horario de la tarde, entre las 12 y las 20 horas del segundo día de pronóstico. Las mayores desviaciones tienen lugar mayormente en la mitad occidental del país, con valores que se enmarcan entre 2,5 y $5 \mathrm{~mm}$. El mayor error se localiza en la zona montañosa del sur de la región central. En la figura 6 se observa que el modelo subestima la magnitud de la precipitación 


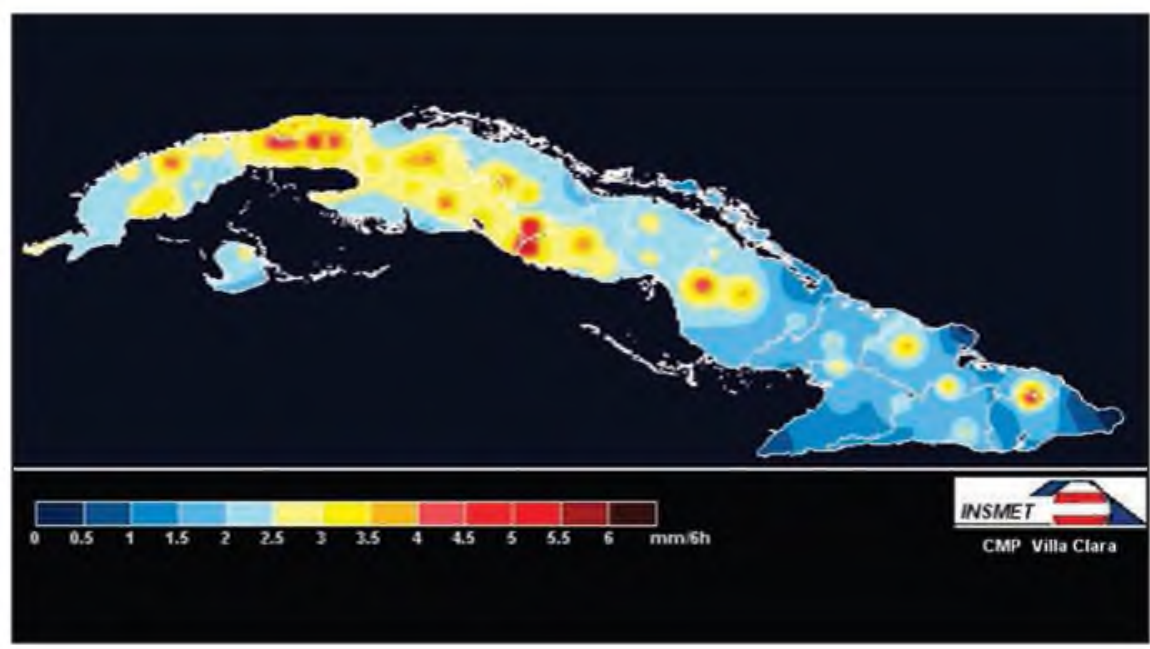

Figura $N^{\circ} 7$ : Campo de la desviación absoluta media del pronóstico para $06-12$ horas (tarde del primer día de pronóstico) respecto al real.

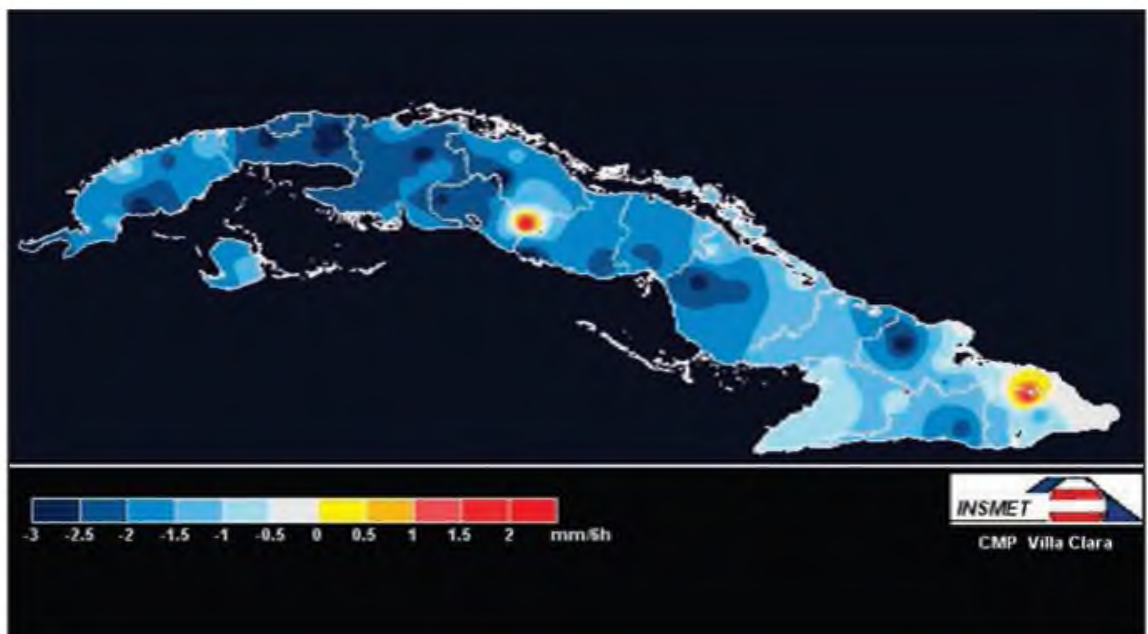

Figura $N^{\circ}$ 8: Campo de la desviación media del pronóstico para 06 - 12 horas (tarde del primer día de pronóstico) respecto al real.

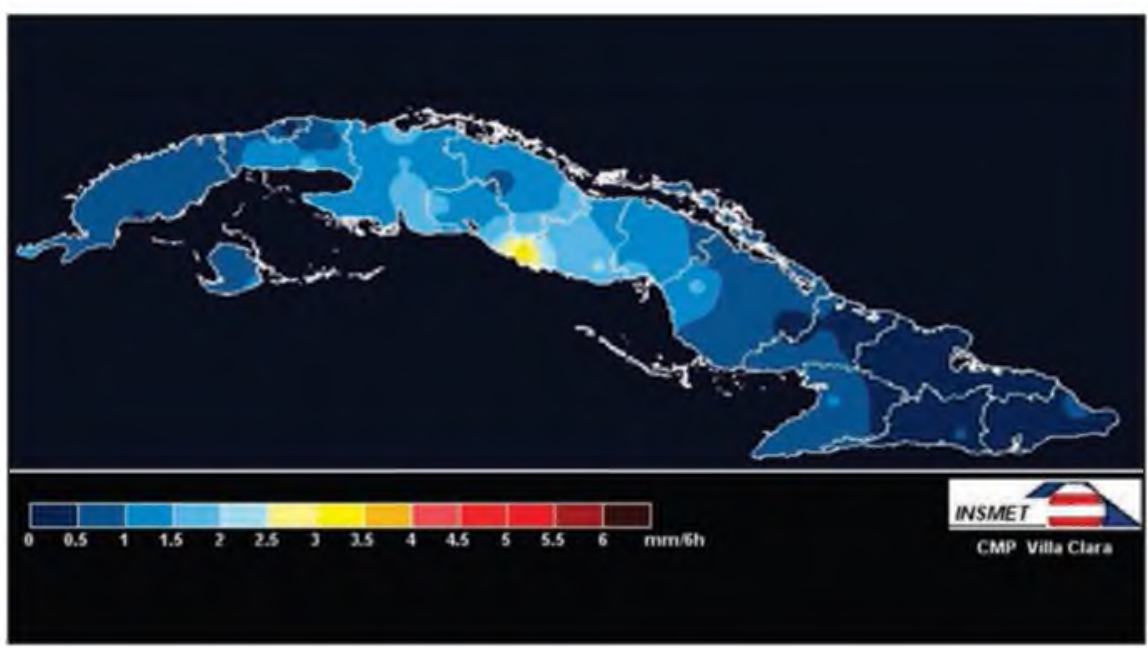

Figura № 9: Campo de la desviación absoluta media del pronóstico para 36 - 42 horas (noche del segundo día de pronóstico) respecto al real. 


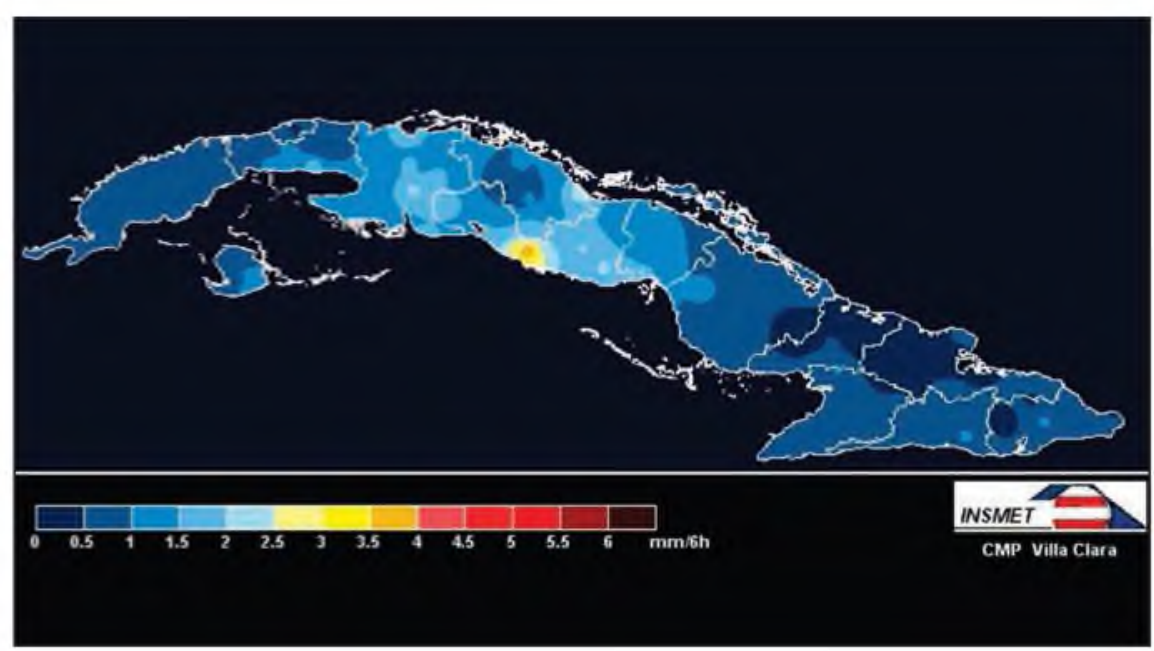

Figura $\mathrm{N}^{\circ}$ 10: Campo de la desviación absoluta media del pronóstico para 12 - 18 horas (noche del primer día de pronóstico) respecto al real.

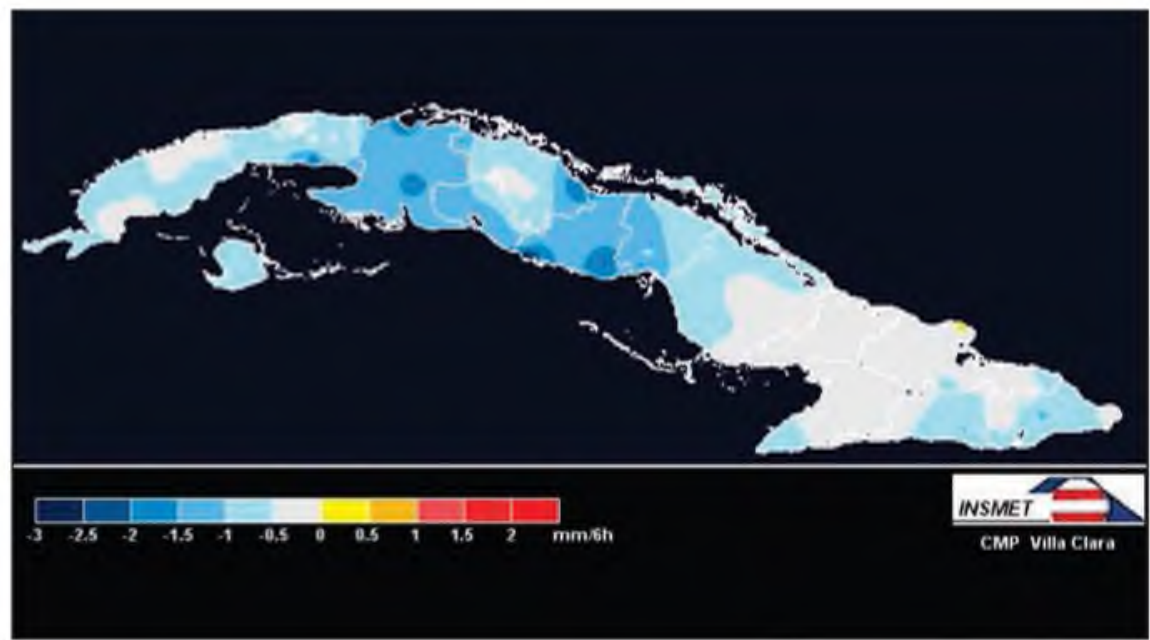

Figura N 11: Campo de la desviación media (sesgo) del pronóstico para 12 - 18 horas (noche del primer día de pronóstico) respecto al real.

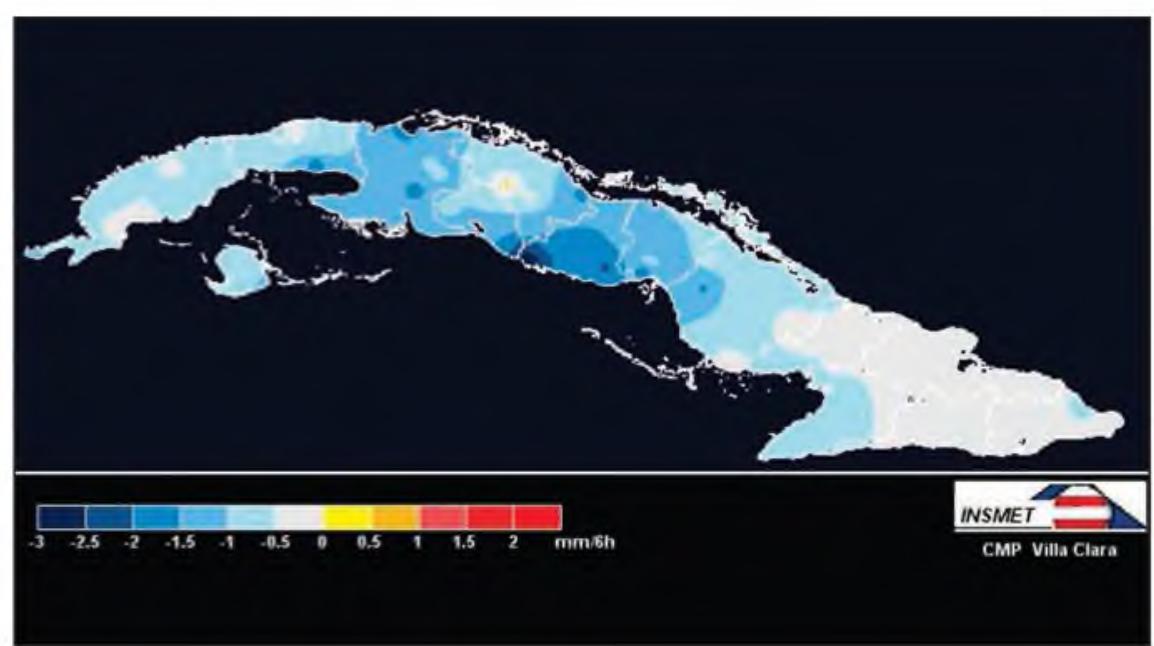

Figura $N^{\circ}$ 12: Campo de la desviación media (sesgo) del pronóstico para 36 - 42 horas (noche del primer día de pronóstico) respecto al real. 
Tabla $N^{\circ} 4$ : İndices estadísticos obtenidos a partir de las tablas de contingencia.

\begin{tabular}{cccccccc}
\hline & \multicolumn{9}{c}{ Indices estadísticos } \\
\hline Plazos & POD & SR & FAR & BIAS & ILL & CN & Efectividad \\
\hline P_06- & 0,81 & 0,86 & 0,14 & 0,95 & 0,55 & 0,79 & 0,82 \\
P_12- & 0,66 & 0,81 & 0,19 & 0,83 & 0,27 & 0,88 & 0,78 \\
P_30- & 0,73 & 0,85 & 0,15 & 0,84 & 0,55 & 0,79 & 0,79 \\
\hline
\end{tabular}

en todo el archipiélago, excepto, en el extremo más oriental.

Gran interés suscitan los resultados alcanzados al evaluar el pronóstico para el plazo 06 - 12 horas, correspondiente a la tarde del primer día de pronóstico (figura $\mathrm{N}^{\circ}$ 7). Aquí se aprecia que los resultados son similares, aunque en algunos puntos las desviaciones absolutas medias son superiores a las observadas en el plazo de pronóstico $30-36$ horas. El error medio total para el plazo 06 - 12 horas fue de 1,91 mm y en el plazo $30-36$ horas fue de 2,3 mm. En este caso se destaca igualmente el macizo montañoso ubicado en el sur de la región central. Al observar la figura $\mathrm{N}^{\circ}$ 8 apreciamos que en este horario el modelo también subestima, excepto en dos puntos, ambos montañosos, que se corresponden con las localidades de La Piedra, en la región Central y Mayarí, en el Oriente del país.

La figura $N^{\circ} 9$ representa los resultados obtenidos para el plazo correspondiente a la noche del segundo día de pronóstico (Pronóstico 36 - 42 h). En este caso las desviaciones medias son inferiores y se concentran en la región central de Cuba, con un núcleo máximo en el macizo montañoso ubicado en el sur de esta región. En este plazo se detectó ocurrencia de precipitación en el $23 \%$ de las celdas tomadas en cuenta durante todos los días considerados. Las máximas desviaciones absolutas en este plazo oscilan entre 1 y $2 \mathrm{~mm}$, excepto en la zona montañosa de la región central, donde alcanzan los $4 \mathrm{~mm}$.

Las desviaciones obtenidas para el período de la madrugada en sentido general son muy inferiores a las del resto de los plazos, por lo que no se reflejan en este documento.

Para el plazo 12 - 18 horas (figura $N^{\circ} 10$ ), correspondiente a la noche del primer día de pronóstico, se obtuvieron resultados similares. En las figuras $N^{\circ} 11,12$ se aprecia que en ambos plazos el modelo subestima la magnitud de la precipitación, fundamentalmente, en el plazo 36 - $42 \mathrm{~h}$ en el sur de la región central del país.

\section{Verificación parcial}

La tabla $N^{\circ} 4$ muestra los índices estadísticos obtenidos a partir de la verificación parcial, que se realizó adoptando el umbral de 0,5 mm como criterio de ocurrencia de lluvia, tanto para el pronóstico, como para la observación.

En la tabla $N^{\circ} 4$ se aprecia que el modelo logra niveles de detección de $81 \%$ en el plazo de pronóstico 06 - 12 y $73 \%$ en el plazo 30 - 36 h. En esta época del año los mayores niveles de precipitación tienen lugar en el período comprendido entre la tarde y las primeras horas de la noche. Obsérvese que se detectó ocurrencia de lluvia (indice ILL) en el $55 \%$ de los casos. En el período de la noche el índice de detección (POD) es inferior y llega hasta el $66 \%$. El índice "BIAS" indica que el modelo subestima en un $19 \%$ los sucesos de

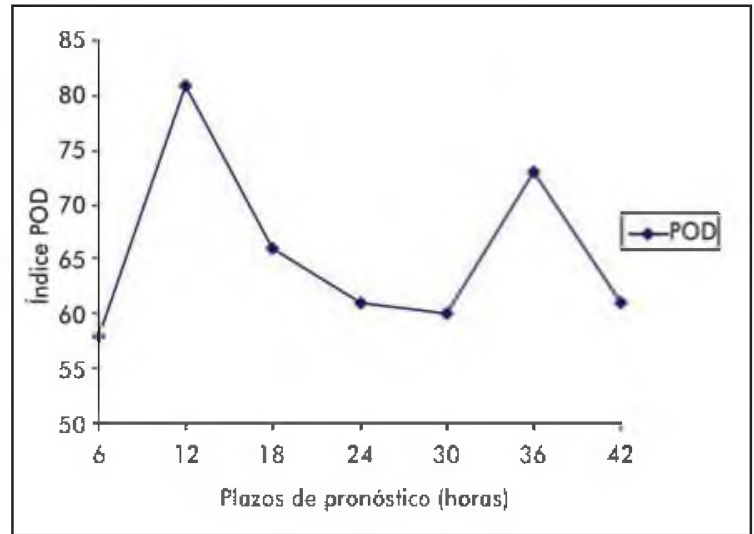

Figura $N^{\circ}$ 13: Comportamiento del índice para los plazos de pronóstico desde 06 hasta 42 horas.

precipitaciones en las noches.

En el período de la tarde el modelo subestima en 5 $\%$ en el plazo de pronóstico 06 - 12 horas y en $16 \%$ en el plazo $30-36$ horas. Se aprecia que, desde el punto de vista de la detección, el modelo es menos efectivo en los plazos correspondientes a las noches, sin embargo, es importante tener en cuenta que estos casos se corresponden con acumulados de precipitaciones poco significativos y en consecuencia, con bajas desviaciones de los valores pronosticados respecto a los observados, como se pudo apreciar en las figuras $N^{\circ} 9,10$. Las figuras $N^{\circ} 13,14$ muestran el comportamiento de los índices y para diferentes plazos de pronóstico. El índice general de efectividad 


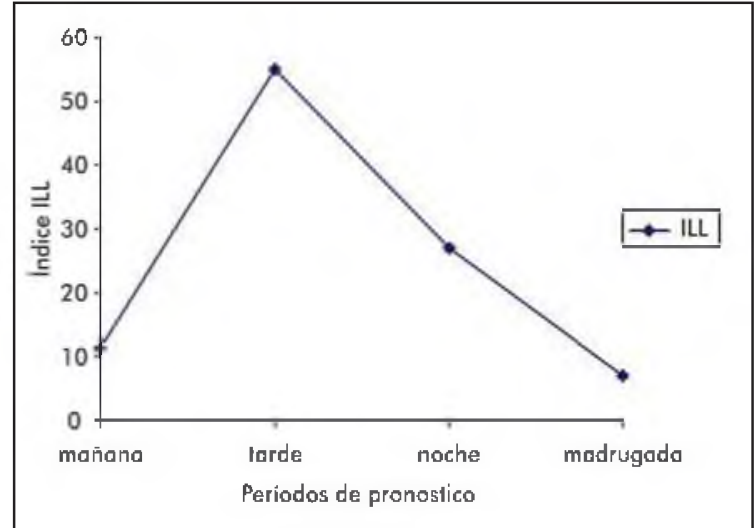

Figura $N^{0}$ 14: Comportamiento del índice para los diferentes plazos del día.

del modelo resultó de $82 \%$ en el período de la tarde del primer día de pronóstico y $78 \%$ en el período de la noche.

\section{DISCUSIÓN}

A partir de la verificación realizada se pudo apreciar que el modelo de forma general subestima la magnitud de las precipitaciones, pero las desviaciones absolutas medias son poco significativas en las tardes, que en definitiva es cuando se producen las precipitaciones más importantes del año en Cuba (excepto en ciclones tropicales). En tanto, el modelo mantiene una estabilidad en el pronóstico cuantitativo al incrementar el horizonte de pronóstico, al menos, hasta 42 horas.

El modelo logra altos niveles de detección. En este caso el índice de detección resultó de $81 \%$ en las tardes del primer día de pronóstico. En el período de la noche el WRF subestima las magnitudes y la ocurrencia de lluvias en general. Una de las causas posibles de esta subestimación puede estar relacionada con el hecho de que en un número importante de ocasiones las nubes de precipitación formadas en las tardes desfogan o continúan desfogando en horas de la noche, cuando las condiciones termodinámicas ya no son favorables para la formación de nuevos núcleos de lluvia y en consecuencia el modelo pronostica "no lluvia". El elevado índice de efectividad general del modelo en el período de la noche se debe fundamentalmente al número de casos en los que el modelo pronostica "no lluvia" y ésta no se produce (índice "CN" de la tabla 4).

Otras variantes de WRF han mostrado índices de detección (POD) de $40 \%$ para umbrales pequeños, con un $40 \%$ también de "falsas alarmas" (26). Mercader et al. (6) obtuvo índices POD de entre 62 y $67 \%$ para un umbral inferior de precipitación de $3 \mathrm{~mm}$ y un horizonte de pronóstico de 18 horas. De manera que los índices estadísticos logrados en esta investigación pueden considerarse satisfactorios

De forma general el modelo constituye hoy una herramienta útil para el trabajo de los pronosticadores de todo el país y es una de las dos versiones operativas hoy en Cuba (sobre las otras dos versiones no aparecen publicaciones) en la literatura científica.

Las conclusiones a las que se arribó al analizar los resultados indican que el modelo WRF fue de gran utilidad para el pronóstico de precipitaciones en Cuba durante el período lluvioso del año 2014. De forma general subestima la magnitud de las precipitaciones, pero las desviaciones absolutas medias del pronóstico respecto a las observaciones no sobrepasan los $5 \mathrm{~mm}$ en las tardes, período en que se producen las lluvias más importantes del año en el país. Al incrementar el horizonte de pronóstico, al menos hasta 42 horas, el modelo mantiene estabilidad en el pronóstico cuantitativo. La versión de WRF aplicada logra mayores niveles de detección del evento "lluvia" en los plazos diurnos, $06-12$ y $30-36$ horas, donde llega a alcanzar un índice POD del 81 y del $73 \%$ respectivamente. En el plazo de pronóstico 12 - 18 horas, correspondiente al período de la noche del primer día de pronóstico, el modelo logró niveles de detección inferiores, con un $66 \%$. El índice BIAS indica que el modelo generalmente subestima la ocurrencia del evento "Iluvia", sin embargo la efectividad general resultó de 82 y $78 \%$ en la tarde y la noche del primer día de pronóstico respectivamente y de $79 \%$ en la tarde del segundo día de pronóstico.

\section{REFERENCIAS BIBLIOGRÁFICAS}

1. The Weather Research \& Forecasting Model [Internet]. Boulder: National Center for Atmospheric Research; [Citado el 15 de mayo de 2015]. Modelo meteorológico WRF [1 pantalla]. Disponible en: http://www.wrf-model.org/index. php

2. Klemp JB. Advances in the WRF model for convection- resolving forecasting, Advances in Geosciences. 2006; 7: 25 - 29.

3. Mitrani I, Alvarez L, Borrajero I. Aplicación optimizada del WRFV3 sobre el territorio cubano mediante el uso de computadora personal, Revista Cubana de Meteorología. 2003; 10 (1): 84 - 94.

4. Turtos L, Capote G, Fonseca $Y$, Alvarez L, Sanchez $M$, Bezanilla $A$, et al. Assessment of the Weather Research and Forecasting model implementation in Cuba addressed to diagnostic air quality modeling. Atmospheric Pollution Research. 2013; 4: $64-74$.

5. Wisse J, Vila'-Guerau de Arellano J. Analysis of the role of the planetary boundary layer schemes 
during a severe convective storm, Ann Geophys. 2004; 22: 1861-1874.

6. Mercader J, Codina B, Sairouni A, Cunillera J. Resultados del modelo meteorológico WRF - ARW sobre Cataluña, utilizando diferentes parametrizaciones de la convección y la microfísica de nubes. Ravista Tethys. 2010; 7: 77 - 89.

7. Kuo HL. On formation and intensification of tropical cyclones through latent heat release by cumulus convection». J. Atmos. Sci. 1965; 22: 4063.

8. Kuo HL. Further studies of the parameterization of the influence of cumulus convection on large-scale flow». J. Atmos. Sci. 1965; 31: 1232-1240.

9. Betts AK, Miller MJ. A new convective adjustement scheme». ECMWF Technical Report. 1984; 43 65.

10. Arakawa A, Schubert WH. Interaction of a cumulus cloud ensemble with the large-scale environment", part I. J. Atmos. Sci. 1974; 31: 674 -701.

11. Grell G, Devenyi DA. Generalized approach to parameterizing convection combining ensemble and data assimilation techniques. Geophysical Research Letters. 2002; 29 (14): 38 - 51.

12. Pleim J. Combined Local and Non local Closure Model for the Atmospheric Boundary Layer. Part I: Model Description and Testing. Journal of Applied Meteorology and Climatology. 2007a; 46 (9), 1383 - 1395

13. Iacono MJ, Delamere JS, Mlawer EJ, Shephard MW, Clough SA, Collins WD. Radiative forcing by long-lived greenhouse gases: Calculations with the AER radiative transfer models. Journal of Geophysical Research: Atmospheres. 2008; 113 (13).

14. Mlawer ES, Taubman $P$, Brown M, lacono MJ, Clough S. Radiative transfer for inhomogeneous atmospheres: RRTM, a validated correlated-k model for the longwave. Journal of Geophysical Research, 1997; 102 (14).

15. Goody RM, Yung YL. Atmospheric Radiation: Theoretical Basis. Oxford University Press. 1995.

16. Lin YL, Farley RD, Orville HD. Bulk parameterization of the snow field in a cloud model, J. Appl. Meteor. 1983; 22, 1065-1092.

17. Pan H, Mahrt L. Interaction between soil hydrology and boundary-layer development. Boundary-Layer Meteorology. 1987; 38 (1): 185 - 202.

18. Chen F. Modeling of land surface evaporation by four schemes and comparison with FIFE observations. Journal of Geophysical Research. D. Atmospheres 1996; 101: 7251 - 7268.

19. Chen F. Cabauw experimental results from the project for intercomparison of land-surface parameterization schemes. Journal of Climate. 1997; $10(6): 1194-1215$.

20. Chen F, Dudhia J. Coupling an advanced land surface-hydrology model with the Penn StateNCAR MM 5 Modeling System. II- Preliminary model validation. Monthly Weather Review. $2001 a ; 129$ (4): $587-604$.

21. Chen F, Dudhia J. 2001 b: Coupling an advanced land surface-hydrology model with the Penn State-NCAR MM5 modeling system. Part I: Model implementation and sensitivity. Monthly Weather Review. 2001 b; 129 (4): 569 - 585.

22. Gordon WJ, Wixon JA. Shepards methodof "metric interpolation" to bivariate and multivariate interpolation. Mathematics of Computation. 1978; 32 (141): $253-264$.

23. Ebert EE. Fuzzy verification of high-resolution gridded forecasts: a review and proposed framework, Meteorol Appl. 2008; 15: 51-64.

24. Wilks DS. Statistical Methods in the Atmospheric Sciences: An Introduction, Academic Press. 1995: $467 \mathrm{pp}$.

25. METEO WEB [Internet]. Santa Clara: Servicio Meteorológico de Villa Clara; [Citado el 2 de junio de 2015]. WRF_Precipitaciones Dom.Cuba [1 pantalla]. Disponible en: http://www.cmp.vcl.cu/ producto/show/codproducto/000050

26. Dillon ME, Skabar YG, Nicolini M. Desempeño del pronóstico de modelos de alta resolución, en un área limitada: análisis de la estación de verano 2010-2011. 2013; 38 (2). 